\title{
On the kinetic and thermodynamic electron temperatures in non-thermal plasmas
}

\author{
R. Alvarez ${ }^{1(a)}$, J. Cotrino ${ }^{1,2}$ and A. Palmero ${ }^{1}$ \\ 1 Instituto de Ciencia de Materiales de Sevilla (CSIC-US) - Americo Vespucio 49, 41092 Seville, Spain \\ 2 Departamento de Física Atómica, Molecular y Nuclear, Universidad de Sevilla - Avda. Reina Mercedes s/n, \\ 41071 Seville, Spain
}

received 2 August 2013; accepted in final form 23 December 2013

published online 27 January 2014

PACS 52.25.Kn - Thermodynamics of plasmas

PACS 52.20. $-j$ - Elementary processes in plasmas

PACS 34.80.Dp - Atomic excitation and ionization

\begin{abstract}
The framework to describe the out-of-equilibrium free electrons in cold plasmas is developed assuming the electron entropy is defined through the Boltzmann H-theorem. Our theory explains why the Saha-Boltzmann relation among higher-lying excited states by means of the electron kinetic temperature is fulfilled, even when free electrons are far from equilibrium. The thermodynamic electron temperature, pressure and chemical potential have been introduced through the derivatives of the electron entropy. It is demonstrated that under usual conditions in cold plasmas, e.g. when the electron distribution function possesses the Maxwellian, Druyvestein or Kappa functional forms, kinetic and thermodynamic electron temperatures yield the same value.
\end{abstract}

Copyright (c) EPLA, 2014

The understanding and characterization of non-thermal plasmas will be without doubts one of the most relevant problems in the next decades. Their continuous appearance in space science, nuclear fusion, nanotechnology or biomedical applications (see, for instance, [1-7]), among others, is posing numerous challenges concerning their dynamics and properties. Unlike thermal plasmas, which may be considered at local thermodynamic equilibrium (LTE), non-thermal plasmas behave as multiple interacting fluids with different dynamics: heavy neutral particles are usually assumed in LTE whereas free electrons are far from LTE and described by means of the Boltzmann kinetic equation [8]. Both fluids, heavy neutral particles and electrons, interact through elastic and inelastic processes that affect the bounded electron density in excited states, which also deviate from LTE. A common quantity to describe the free electron population is the so-called electron kinetic temperature, $T_{e}$, whose definition is extrapolated from a LTE relation

$$
k_{B} T_{e}=\frac{2}{3} \varepsilon
$$

where $k_{B}$ is the Boltzmann constant and $\varepsilon$ the electron mean kinetic energy. Equation (1) contrasts with the

\footnotetext{
(a) E-mail: rafael.alvarez@icmse.csic.es
}

definition of temperature made through thermodynamic principles and related to the changes of entropy with energy. In fact, the thermodynamic definition of temperature is inherently connected to the concept of thermal equilibrium, by which two interacting systems reach a steady state defined by a common value of their temperatures. Since eq. (1) extrapolates a LTE relation for a non-LTE system, the kinetic temperature, as such, should be understood as a measure of $\varepsilon$ with no thermodynamic implications.

Despite the considerations above, theoretical and experimental evidences in the literature show that there is an ionization/recombination balance between the (out-ofequilibrium) free electrons and higher-lying excited states of neutral particles defined by the electron kinetic temperature: more than two decades ago, Fujimoto et al. [9] and van der Mullen [10] published two seminal works about the thermodynamic equilibrium in non-thermal hydrogenlike plasmas. These works pointed out that higher-lying excited states, defined by an effective quantum number, $p$, fulfilled the relation

$$
\frac{n_{p}}{n_{p}^{S B}} \simeq 1+b_{0} p^{-x}
$$

where $n_{p}$ is the actual density of excited particles, $b_{0}$ a constant, $x \sim 6$ and $n_{p}^{S B}$ the excited state population density 
value obtained through the so-called Saha-Boltzmann relation, which corresponds to a LTE collisional ionizationrecombination balance

$$
\frac{n_{p}^{S B}}{n_{e}^{2}}=\frac{g_{p}}{2 g_{+}} \frac{h^{3}}{\left(2 \pi m_{e} k_{B} T_{e}\right)^{3 / 2}} \exp \left(\frac{E_{p \lambda}}{k_{B} T_{e}}\right) .
$$

In eq. (3), $g_{p}$ and $g_{+}$are the statistical weight of level $p$ and that of the ion in the ground level, respectively, $m_{e}$ is the electron mass, $E_{p \lambda}$ the ionization energy from level $p$ and $n_{e}$ the electron density. Equation (2) points out that for high values of $p$, the relation $\left(n_{p} / n_{p}^{S B}\right)_{p \gg} \simeq 1$ is fulfilled, i.e., the population density of upper excited atomic states follows a LTE relation defined by the electron kinetic temperature. Also, using eq. (3), the ratio between the populations of two upper excited levels, $p$ and $p^{\prime}$ is

$$
\left(\frac{n_{p}}{n_{p^{\prime}}}\right)_{p, p^{\prime} \gg} \simeq \frac{g_{p}}{g_{p^{\prime}}} \exp \left(\frac{E_{p \lambda}-E_{p^{\prime} \lambda}}{k_{B} T_{e}}\right),
$$

i.e., the Boltzmann LTE relation between levels at temperature $T_{e}$. Although Fujimoto and van der Mullen found this result assuming a Maxwellian electron distribution function (EDF), similar results have been obtained in situations where the EDFs were far from the Maxwellian functional form. In ref. [11], the homogeneous Boltzmann equation for the free electrons was solved using the two terms Legendre expansion approximation [8], finding again eq. (2) with $x$ between 5.5 and 6.5 .

From an experimental point of view, there are numerous evidences of the above-mentioned ionizationrecombination balance with free electrons, mostly thanks to optical emission spectroscopy data collected from radiative decays of upper excited states of neutral particles in plasmas produced at the laboratory: in ref. [12], for instance, a microwave argon discharge at atmospheric pressure was analyzed finding that the relation $\left(n_{p} / n_{p}^{S B}\right) \simeq 1$ holds when $p>3$. Also, in ref. [13] the thermal inequilibrium of a non-thermal, atmospheric helium microwave plasma was studied through optical emission spectroscopy, obtaining that the population density of neutral particles excited states also followed eq. (2) with $b_{0} \sim 10^{4}$ and $x \sim 6-7$. Thus, eqs. (3), (4) were fulfilled for higherlying excited levels (i.e., $p \geq 7$ ). This also agrees with ref. [14], where a helium plasma jet was studied, and where it is concluded that an ionization/recombination balance between free electrons and upper excited states was established. Moreover, a non-thermal plasma of mercury is analyzed in ref. [15], finding that the population density of the excited states above the level $6^{3} D_{3}$ fulfills the relation $\left(n_{p} / n_{p}^{S B}\right) \simeq 1$. These are results from some selected papers in the literature, but there are numerous evidences of this behavior in many situations (see, for instance, refs. [12-17] and references therein). The existence of this balance is explained by considering the high interaction rate between free electrons and higherlying excited states, caused by the low-energy threshold for electron-induced collisional ionization/recombination processes [18]. Lower-bounded excited levels, on the other hand, deviate from this balance because direct electroninduced ionization processes do not dominate over other radiative, diffusive or excitation processes.

The experimental evidence described above poses different fundamental questions on the behavior of cold plasmas, and particularly:

1) How may a system out of equilibrium (free electrons) impose a typical LTE balance over higher-lying excited levels of neutral particles?

2) Why is such LTE balance governed by the electron kinetic temperature?

Indeed, the existence of a Saha-Boltzmann balance between excited levels by means of the electron kinetic temperature, eqs. (3), (4), for so many different cold plasmas, with different chemical composition and when electrons are out of equilibrium, strongly suggest that this balance emerges thanks to a general thermodynamic principle valid for equilibrium and non-equilibrium systems. The concept of thermodynamic quantities for nonLTE systems has been widely discussed in the literature in many situations [19-21], and is still an open problem of the outmost relevance for general systems far from equilibrium [22]. In this letter we develop a theory to study the interaction of free electrons with other systems through the definition of entropy given by the Boltzmann H-theorem. Our theory successfully explains why the Saha-Boltzmann relation still holds when electrons are out of equilibrium, and introduces new quantities, such as the thermodynamic temperature or thermodynamic chemical potential, that help describe the non-equilibrium free electrons in a general out-of-equilibrium framework.

We call $F(t, \vec{r}, \vec{v} \mid \vec{\chi})$ the electron distribution function, where $t$ represents time, $\vec{r}$ the position vector, $\vec{v}$ the velocity vector and $\vec{\chi} \equiv\left(\chi_{1}, \chi_{2}, \cdots\right)$ the set of parameters that determines the shape of the EDF (for instance, the local value of the electric field, electron density, ionization degree, etc.). We introduce the entropy of the free electrons, $S_{e}$, as

$$
\begin{aligned}
S_{e}(t \mid \vec{\chi})= & -k_{B} \int \mathrm{d}^{3} r \mathrm{~d}^{3} v F(t, \vec{r}, \vec{v} \mid \vec{\chi}) \ln F(t, \vec{r}, \vec{v} \mid \vec{\chi}) \\
& +N_{e} s_{0},
\end{aligned}
$$

where $N_{e}$ is the number of electrons. This quantity is known to fulfill all the requirements for an entropy whenever the Boltzmann equation is applicable $\left(S_{e}\right.$ is additive for several systems, it monotonically increases in time and yields well-known equilibrium relations). In order to simplify our calculations we assume a steady-state situation and a homogeneous distribution of electrons: $F(t, \vec{r}, \vec{v} \mid \vec{\chi}) \equiv n_{e} f(\vec{v} \mid \vec{\chi})$, with $f(\vec{v} \mid \vec{\chi})$ the velocity EDF normalized as $\int \mathrm{d}^{3} v f(\vec{v} \mid \vec{\chi})=1$, and $\int \mathrm{d}^{3} r n_{e}=N_{e}$. In this way, eq. (5) turns into

$$
S_{e}=-N_{e} k_{B} \ln \frac{N_{e}}{V_{e}}+N_{e} k_{B} \tilde{s}(\vec{\chi})+N_{e} s_{0}
$$


with $V_{e}$ the volume of the electron system and $\tilde{s}(\vec{\chi})=$ $-\int \mathrm{d}^{3} v f(\vec{v} \mid \vec{\chi}) \ln f(\vec{v} \mid \vec{\chi})$. Due to the relevance of $\tilde{s}$ in our theory, we have called it CAP-entropy in this letter. The energy of the electrons, $U_{e}$, is introduced as

$$
U_{e}=\int \mathrm{d}^{3} r \mathrm{~d}^{3} v F(t, \vec{r}, \vec{v} \mid \vec{\chi}) \frac{1}{2} m_{e} v^{2}=N_{e} \varepsilon,
$$

where $\varepsilon=\int \mathrm{d}^{3} v \frac{1}{2} m_{e} v^{2} f(\vec{v} \mid \vec{\chi})$ is the above-mentioned electron mean kinetic energy. Given eqs. (6), (7), and following ref. [20], we introduce the thermodynamic quantities for the electron system as an extension of the Gibbs relation:

$$
\mathrm{d} S_{e}=\frac{\mathrm{d} U_{e}}{T_{e}^{t h}}+\frac{p_{e}^{t h}}{T_{e}^{t h}} \mathrm{~d} V_{e}-\frac{\mu_{e}^{t h}}{T_{e}^{t h}} \mathrm{~d} N_{e},
$$

with $T_{e}^{t h}$ being the thermodynamic electron temperature, $p_{e}^{t h}$ the electron thermodynamic pressure and $\mu_{e}^{t h}$ the electron thermodynamic chemical potential. Using eqs. (6)-(8) and making the derivatives of the entropy with respect to $U_{e}$ we find

$$
\frac{1}{k_{B} T_{e}^{t h}}=\left(\frac{\partial \tilde{s}}{\partial \varepsilon}\right)_{N_{e}, V_{e}}
$$

and calculating the derivative of the entropy with respect to $V_{e}$,

$$
\frac{p_{e}^{t h}}{n_{e} k_{B} T_{e}^{t h}}=1-n_{e}\left(\frac{\partial \tilde{s}}{\partial n_{e}}\right)_{\varepsilon, N_{e}},
$$

which is the free electrons equation of state. However, given the relation between internal energy and kinetic energy of the electrons in eq. (7), we expect that the condition $n_{e}\left(\partial \tilde{s} / \partial n_{e}\right)_{\varepsilon, N_{e}} \ll 1$ is always fulfilled in practical cases. Finally, the partial derivative of $S_{e}$ with respect to $N_{e}$ obtains the well-known Euler relation for extensive and intensive quantities $S_{e}=\left(U_{e} / T_{e}^{t h}\right)+\left(p_{e}^{t h} V_{e} / T_{e}^{t h}\right)-$ $\left(\mu_{e}^{t h} N_{e} / T_{e}^{t h}\right)$ which, taking into account eq. (8), results in the well-known Gibbs-Duhem relation [23]

$$
U_{e} \mathrm{~d}\left(\frac{1}{T_{e}^{t h}}\right)+V_{e} \mathrm{~d}\left(\frac{p_{e}^{t h}}{T_{e}^{t h}}\right)-N_{e} \mathrm{~d}\left(\frac{\mu_{e}^{t h}}{T_{e}^{t h}}\right)=0 .
$$

The definition of electron temperature through the CAPentropy in eq. (9) clearly differs with that in eq. (1), and in general, different values are expected. However, there are cases where both definitions of electron temperature yield the same value: for instance, if $f(\vec{v} \mid \vec{\chi})$ only depends on the velocity modulus, $v$, and there is a system parameter, $\chi_{1}$, through which $f(v \mid \vec{\chi})$ can be written as

$$
f(\vec{v} \mid \vec{\chi}) \equiv \chi_{1}^{-3} g\left(\frac{v}{\chi_{1}} \mid \chi_{2} \ldots\right)
$$

it is found that $T_{e}=T_{e}^{t h}$ (in the appendix we show an original demonstration of this statement). Remarkably, the form of the EDF in eq. (12) is widely employed in the description of the free electron population in non-thermal plasmas: for instance, the Maxwellian or the Druyvestein
EDFs, as well as other fitting expressions employed in the literature for laboratory plasmas [24]. Furthermore, the well-known Kappa distribution [25], widely used in space plasmas, also possesses same mathematical form. Therefore, in all these cases, the relation $T_{e}=T_{e}^{t h}$ holds.

In general, the EDF does not have the form given in eq. (12). In order to check the relation between both temperatures in a more general context, we have solved the homogeneous steady-state Boltzmann equation in the two terms Legendre expansion approximation [8] using the software BOLSIG $[26,27]$. This software is well accepted and tested in the literature for non-thermal plasmas produced in laboratory, and has been successfully applied in many cases for non-magnetized plasmas (see for instance [27-29]). If electron-electron interactions are considered, the input quantities in BOLSIG are $\vec{\chi}=\left(E / N, N, n_{e} / N\right)$, where $E$ is the value of the effective local electric field and $N$ the density of neutral particles. Here, we have to point out that, strictly speaking, regular collisional-radiative models require a self-consistent coupling of the Boltzmann equation with a kinetic model, and therefore, the particular values of $E, N$ and $n_{e}$ would be imposed in each problem based on the value of different input experimentally controllable quantities (e.g., external electromagnetic field, condition of the gases, geometrical parameters, etc.). We have systematically solved the software BOLSIG in nearly 20000 conditions for plasmas with different chemical compositions and values of $E, N$ and $n_{e}$. However, since the electron-electron term only considers the isotropic component of the EDF [27,30,31], we have neglected the anisotropic contribution in our calculation for consistency. Furthermore, the relation between $E / N$ and $\varepsilon$ is univocally defined for given values of $N$ and $n_{e} / N$, thus, for clarity purposes, we present the results as a function of $N, n_{e} / N$ and $\varepsilon$. In figs. 1(a)-(e), we show the typical shape of some EDFs calculated to the zeroth order in the Legendre expansion for $\mathrm{Ar}, \mathrm{He}, \mathrm{N}_{2}, \mathrm{O}_{2}$ and $\mathrm{H}_{2}$ plasmas: the base conditions correspond to a plasma with $\varepsilon=4 \mathrm{eV}$, a density of neutrals of $N=2.4 \times 10^{20} \mathrm{~m}^{-3}$ (typical value for a pressure of $1 \mathrm{~Pa}$ at room temperature) and an ionization degree of $n_{e} / N=10^{-4}$, which is depicted with a solid line. The rest of the EDFs was calculated by varying one parameter at a time. Figures 1(a)-(e) illustrate that the shapes of the EDFs are clearly far from the Maxwellian profile in most of the cases (note that the Maxwellian profile is a straight line in the chosen representation), being the particular dependence of the EDF on the input parameters explained elsewhere [27]. In figs. 1(f)-(j) we present the value of $\tilde{s}$ as a function of $\varepsilon$ for the five chemical compositions of the plasmas under study (Ar, $\mathrm{He}, \mathrm{N}_{2}, \mathrm{O}_{2}$ and $\mathrm{H}_{2}$ ), and for given values of $N$ and $n_{e} / N$ : three different neutral particles densities $N=2.4 \times 10^{20} \mathrm{~m}^{-3}$ (typical for a gas pressure of $1 \mathrm{~Pa}$ at room temperature), $N=3.2 \times 10^{22} \mathrm{~m}^{-3}$ (typical for a gas pressure of 1 torr $=133 \mathrm{~Pa}$ at room temperature), and $N=3.2 \times 10^{24} \mathrm{~m}^{-3}$ (typical for a gas pressure of 100 torr $=1.3 \times 10^{4} \mathrm{~Pa}$ at room temperature), ionization 


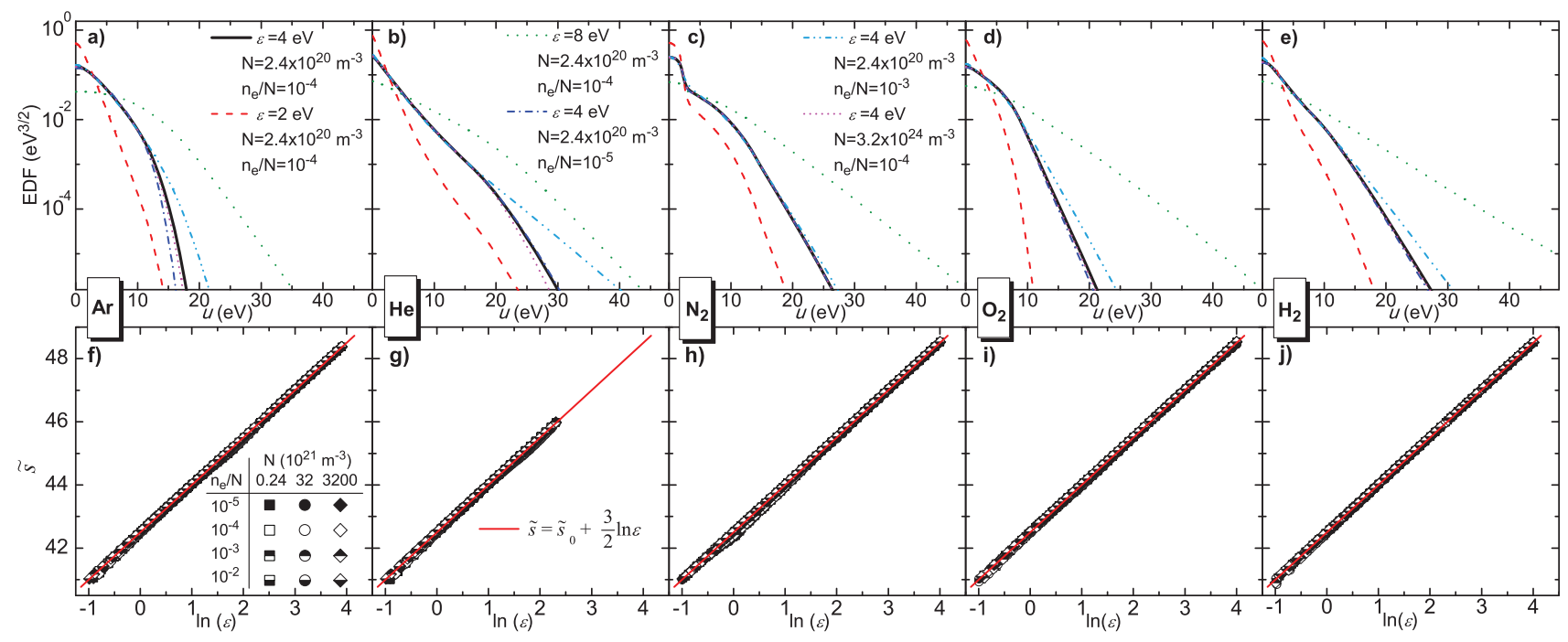

Fig. 1: (Colour on-line) Shape of some calculated EDFs for (a) Ar, (b) He, (c) $\mathrm{N}_{2}$, (d) $\mathrm{O}_{2}$ and (e) $\mathrm{H}_{2}$ plasmas: the base condition corresponds to a mean kinetic energy of $\varepsilon=4 \mathrm{eV}$, a density of neutrals of $N=2.4 \times 10^{20} \mathrm{~m}^{-3}$ (typical value for a pressure of $1 \mathrm{~Pa}$ at room temperature) and an ionization degree of $n_{e} / N=10^{-4}$, which is depicted with a solid line. The rest of the EDFs was calculated by varying a single parameter. Note that the Maxwellian (equilibrium) profile should be depicted as a straight line in the chosen representation. In panels (f)-(j) we present the value of $\tilde{s}$ as a function of $\varepsilon$, for given values of $N$ and $n_{e} / N$ and for (f) Ar, (g) $\mathrm{He},(\mathrm{h}) \mathrm{N}_{2}$, (i) $\mathrm{O}_{2}$ and (f) $\mathrm{H}_{2}$ plasmas. We present results for three different neutral particles densities: $N=2.4 \times 10^{20} \mathrm{~m}^{-3}$ (typical for a gas pressure of $1 \mathrm{~Pa}$ at room temperature), $N=3.2 \times 10^{22} \mathrm{~m}^{-3}$ (typical for a gas pressure of 1 torr $=133 \mathrm{~Pa}$ at room temperature), and $N=3.2 \times 10^{24} \mathrm{~m}^{-3}$ (typical for a gas pressure of 100 torr $=1.3 \times 10^{4} \mathrm{~Pa}$ at room temperature), for ionization degrees ranging from $n_{e} / N=10^{-2}$ to $n_{e} / N=10^{-5}$, and values of $\varepsilon$ ranging from $0.3 \mathrm{eV}$ to $55 \mathrm{eV}$ for all the cases, except for He for which we only found convergence of the calculations up to $\varepsilon=11 \mathrm{eV}$.

degrees ranging from $n_{e} / N=10^{-2}$ to $n_{e} / N=10^{-5}$, and values of $\varepsilon$ ranging from $0.3 \mathrm{eV}$ to $55 \mathrm{eV}$ (except in the case of helium, where we only found convergence of the BOLSIG calculations up to $\varepsilon=11 \mathrm{eV}$ ). We constrained our study within these ranges in order to have typical values in non-thermal plasmas produced at the laboratory and typical astronomical plasmas. It is worthy to mention that, strictly speaking, the EDF of molecular gases must be calculated by considering the vibrational distribution function of the ground state of the molecules, as well as any possible dissociation mechanism [32,33], processes that BOLSIG does not take into account. Therefore, our results may include some inaccuracies due to these facts. The first remarkable result is the very weak dependence between $\tilde{s}$ and $n_{e}$ for a given value of $\varepsilon$ : in all the studied conditions, the quantity $n_{e}\left(\partial \tilde{s} / \partial n_{e}\right)_{\varepsilon, V_{e}}$ in eq. (10) is always below $10^{-2}$ and, as expected

$$
\frac{p_{e}^{t h}}{n_{e} k_{B} T_{e}^{t h}} \simeq 1
$$

The second remarkable result is the general trend obtained between $\tilde{s}$ and $\varepsilon$, as it accurately follows the relation $\tilde{s}=\tilde{s}_{0}+\frac{3}{2} \ln \varepsilon$ in all the studied conditions. Hence, using eqs. (1) and (9) we obtain

$$
T_{e}^{t h}=T_{e} .
$$

This indicates that definitions as different as that of $T_{e}$, connected to the mean kinetic energy of free electrons, and that of $T_{e}^{t h}$, linked to the relation between entropy and energy, yield the same values in typical conditions. Making use of eqs. (7), (11), (13) and (14), we find

$$
\mathrm{d}\left(\frac{\mu_{e}^{t h}}{T_{e}}\right)=\frac{3}{2} k_{B} T_{e} \mathrm{~d}\left(\frac{1}{T_{e}}\right)+\frac{k_{B}}{n_{e}} \mathrm{~d} n_{e},
$$

which can be integrated as

$$
\mu_{e}^{t h}=\mu_{e}^{(0)}-\frac{3}{2} k_{B} T_{e} \ln T_{e}+k_{B} T_{e} \ln n_{e},
$$

i.e., the thermodynamic chemical potential of the free electrons has the same form as that of an ideal gas (within the limits of the kinetic theory of gases). Thus, following a parallel deduction to that in ref. [34] (where free electrons are treated as an ideal gas at a temperature equal to the kinetic temperature) the ionization-recombination balance $X_{p}+e \rightleftarrows X^{+}+e+e$, associated to a maximum of entropy between electrons, neutrals and ions, yields the relation

$$
\frac{\mu_{e}^{t h}}{T_{e}}-\frac{\mu_{p}}{T_{g}}+\frac{\mu_{+}}{T_{g}}=0
$$

where $\mu_{p}$ is the chemical potential of neutral particles in the excited state $p, T_{g}$ the gas temperature and $\mu_{+}$ the chemical potential of the ion (we have assumed that they are at LTE and with temperature $T_{q}$ ). In this way, as was demonstrated in ref. [34], eq. (16) together with eq. (15) yields the Saha-Boltzmann equation, eq. (3), and the Boltzmann balance, eq. (4). 
Summarizing, we have obtained the following results:

i) the electron thermodynamic temperature is equal to the electron kinetic temperature under the usual conditions in cold plasmas.

ii) In these conditions, free electrons are well described by a thermodynamic chemical potential with the same mathematical form as that of an ideal gas at the electron kinetic temperature, eq. (15).

iii) A maximum of entropy governing the ionizationrecombination balance, $X_{p}+e \rightleftarrows X^{+}+e+e$, implies eq. (16), and therefore the Saha-Boltzmann balance in eq. (3) and the Boltzmann relation among levels, eq. (4).

The three results above explain the numerous evidences on the existence of higher-lying excited states in cold plasmas that follow the Boltzmann distribution according to the electron kinetic temperature, even when free electrons are out of equilibrium. Of course, when ions or other species play a significant role in the ionization mechanisms of the higher-lying excited states in the plasma, the ionization-recombination balance with free electrons may be disrupted. Indeed, in this letter we have not analyzed why such balance emerges, but rather the validity of eqs. (3), (4) when this balance is induced by out-ofequilibrium electrons. Finally, we want to stress that, although we have deduced our theory for global systems, it can also be extrapolated to local behaviors.

In this letter we have developed a framework to describe the out-of-equilibrium free electrons in cold plasmas that has explained the validity of the Saha-Boltzmann balance among higher-lying excited states by means of the electron kinetic temperature. Moreover, in the course of this investigation, a set of new thermodynamic quantities has been introduced that defines the out-of-equilibrium free electrons and their properties when their interaction with another system is governed by a maximum of entropy.

$$
* * *
$$

We thank the Junta de Andalucía (Projects P12-FQM2265, TEP-08067 and P10-FQM-6900) and the Ministry of Science and Innovation (Projects CONSOLIDER CSD2008-00023, MAT2010-21228 and MAT2010-18447) for financial support.

Appendix: demonstration that $T_{e}=T_{e}^{t h}$ when $f(\vec{v} \mid \vec{\chi}) \equiv \chi_{1}^{-3} g\left(v / \chi_{1} \mid \chi_{2} \cdots\right)$. - When $f(\vec{v} \mid \vec{\chi}) \equiv$ $\chi_{1}^{-3} g\left(v / \chi_{1} \mid \chi_{2} \ldots\right)$, the mean kinetic energy is found to be $\varepsilon \propto \chi_{1}^{2}$, so $f(\vec{v} \mid \vec{\chi})$ can be rewritten as $f(\vec{v} \mid \vec{\chi}) \equiv$ $\varepsilon^{-3 / 2} g^{\prime}\left(v / \sqrt{\varepsilon} \mid \chi_{2} \cdots\right)$ and the relation

$$
\frac{1}{v^{2}}\left[\frac{\partial}{\partial v}\left(v^{3} f\right)\right]_{\varepsilon, \chi_{2}, \cdots}=-2 \varepsilon\left(\frac{\partial f}{\partial \varepsilon}\right)_{v, \chi_{2}, \cdots}
$$

is fulfilled. Using eq. (9), the thermodynamic temperature is calculated as

$$
\begin{aligned}
& \frac{1}{k_{B} T_{e}^{t h}}=-\left(\frac{\partial h}{\partial \varepsilon}\right)_{N_{e}, V_{e}} \\
& =-\int \mathrm{d}^{3} v \ln f\left(\frac{\partial f}{\partial \varepsilon}\right)_{v, N_{e}, V_{e}}-\int \mathrm{d}^{3} v\left(\frac{\partial f}{\partial \varepsilon}\right)_{v, N_{e}, V_{e}} \\
& =-\int \mathrm{d}^{3} v \ln f\left(\frac{\partial f}{\partial \varepsilon}\right)_{v, N_{e}, V_{e}}-\left(\frac{\partial}{\partial \varepsilon}\right)_{N_{e}, V_{e}} \int \mathrm{d}^{3} v f
\end{aligned}
$$

where we have employed the normalization condition $\int \mathrm{d}^{3} v f=1$. Using eq. (A.1) and (A.2) we calculate

$\frac{1}{k_{B} T_{e}^{t h}}=\int \mathrm{d}^{3} v \frac{1}{2 \varepsilon v^{2}} \ln f \frac{\partial\left(f v^{3}\right)}{\partial v}=\frac{2 \pi}{\varepsilon} \int_{0}^{\infty} \mathrm{d} v \ln f \frac{\partial\left(f v^{3}\right)}{\partial v}$

$=\frac{2 \pi}{\varepsilon} \int_{0}^{\infty} \mathrm{d} v\left[\frac{\partial\left(v^{3} f \ln f\right)}{\partial v}-v^{3} \frac{\partial f}{\partial v}\right]=\frac{2 \pi}{\tau \lim _{v \rightarrow \infty}\left(v^{3} f \ln f\right)}$

$-\frac{2 \pi}{\varepsilon} \int_{0}^{\infty} \mathrm{d} v\left[\frac{\partial\left(f v^{3}\right)}{\partial v}-3 f v^{2}\right]=\frac{-\frac{2 \pi}{3} \lim _{v \rightarrow \infty}\left(f v^{3}\right)}{3}$

$+\frac{6 \pi}{\varepsilon} \int_{0}^{\infty} \mathrm{d} v v^{2} f=\frac{3}{2 \varepsilon} \int_{0}^{\infty} \mathrm{d}^{3} v f=\frac{3}{2 \varepsilon}=\frac{1}{k_{B} T_{e}}$,

where we have employed that the EDF is well defined and therefore $\lim _{v \rightarrow \infty}\left(v^{3} f \ln f\right)=0$ and $\lim _{v \rightarrow \infty}\left(f v^{3}\right)=0$.

\section{REFERENCES}

[1] Wahlund J.-E., Boström R., Gustafsson G., GurNETT D. A. et al., Science, 308 (2005) 986.

[2] Burch J. L., Goldstein J., Lewis W. S., Young D. T., Contes A. J., Dougherty M. K. and André N., Nature, 447 (2007) 833.

[3] Rubio S. J., Quintero M. C., Rodero A. and Alvarez R., Acta Phys. Slovaca, 54 (2004) 125.

[4] Alvarez R., Garcia-Martin J. M., Macias-Montero M., Gonzalez-Garcia L., Gonzalez J. C., Rico V., Perlich J., Cotrino J., Gonzalez-Elipe A. R. and Palmero A., Nanotechnology, 24 (2013) 045604.

[5] Blossey R., Nat. Mater., 2 (2003) 301.

[6] Kim G. C., Lee H. W., Byun J. H., Chung J., Jeon Y. C. and Lee J. K., Plasma Process. Polym., 10 (2013) 199.

[7] Ziuzina D., Patil S., Cullen P. J., Keener K. M. and Bourke P., J. Appl. Microbiol., 114 (2012) 778.

[8] Golant V. E., Zhilinsky A. P. and Sakharov I. E., in Fundamentals of Plasma Physics, edited by SANBOrN C. Brown (John Wiley \& Sons) 1990, ISBN:0-47104593-4.

[9] Fujimoto T. and McWhirter R. W. P., Phys. Rev. A, 42 (1990) 6588.

[10] van der Mullen J. A. M., Phys. Rep., 191 (1990) 109. 
[11] Vlcek J. and Pelikán V., J. Phys. D: Appl. Phys., 22 (1989) 632.

[12] Sainz A., Margot J., Garcia M. C. and Calzada D., J. Appl. Phys., 97 (2005) 113305.

[13] Alvarez R., Rodero A., Quintero M. C., Sola A., Gamero A. and Ortega D., J. Appl. Phys., 98 (2005) 093304.

[14] Xiong Q., Nikiforov A. Y., Gonzalez M. A., Leys C. and Lu X. P., Plasma Source Sci. Technol., 22 (2013) 015011.

[15] Karabourniotis D., J. Appl. Phys., 92 (2002) 25.

[16] Cotrino J., Palmero A., Rico V., Barranco A., EsPinos J. P. and González-Elipe A. R., J. Vac. Sci. Technol. B, 19 (2001) 410.

[17] Lao C., Cotrino J., Palmero A., Gamero A. and Gonzalez-Elipe A. R., Eur. Phys. J. D: Appl. Phys., 14 (2001) 361.

[18] Akatsuka H., Phys. Plasmas, 16 (2009) 043502.

[19] Brey J. J. and Santos A., Phys. Rev. A, 45 (1992) 8566.

[20] Narayanan K. R. and Srinivasa A. R., Phys. Rev. E, 85 (2012) 031151.

[21] Lieou C. K. C. and Langer J. S., Phys. Rev. E, 85 (2012) 061308.

[22] Livadiotis G. and Comas D. J., Space Sci. Rev., 175 (2013) 183.
[23] Callen H. B., Thermodynamics and an Introduction of Thermostatistics, second edition (John Willey \& Sons) 1985, ISBN 0-471-86256-8.

[24] Nambu K., Mitsui K. and Kondo S., J. Phys. D: Appl. Phys., 33 (2000) 2274.

[25] Pierrard V. and Lazar M., Sol. Phys., 267 (2010) 153.

[26] http://www.bolsig.laplace.univ-tlse.fr/

[27] Hagelaar G. J. M. and Pitchford L. C., Plasma Sources Sci. Technol., 14 (2005) 722.

[28] Ristic M. M., Vojnovic M., Poparic G. B. and Belic D. S., Chem. Phys., 405 (2012) 16.

[29] Lisovkiy V., Yegorenkov V., Booth J. P., Landry K., Douai D. and Cassagne V., J. Phys. D: Appl. Phys., 43 (2010) 385203.

[30] Rockwood S. D., Phys. Rev. A, 8 (1973) 2348.

[31] Rosenbluth M. N., MacDonald W. M. and Judd D. L., Phys. Rev., 107 (1957) 1.

[32] Guerra V. and Loureiro J., Plasma Sources Sci. Technol., 6 (1997) 373.

[33] Guerra V., Sá P. A. and Loureiro J., Eur. Phys. J. Appl. Phys., 28 (2004) 125.

[34] van der Sanden M. C. M., Schram P. P. J. M., Peeters A. G., van der Mullen J. A. M. and KroeSEN G. M. W., Phys. Rev. A, 40 (1989) 5273. 